\title{
COSTAL CHONDRITIS
}

\author{
R. A. Elson, Oswestry, England \\ From The London Hospital, London
}

Diseases of the skull, facial skeleton and ribs are usually treated by surgeons who are familiar with the vital organs which these bones enclose. Nevertheless, orthopaedic surgeons may be consulted when any musculo-skeletal tissue is diseased and so should be familiar with specific conditions peculiar to the part. Infection of the costal cartilages (suppurative chondritis, necrotising chondritis) is such a condition.

Costal chondritis is a well defined entity. It shows how basic surgical principles are little altered by modern chemotherapeutic advances; the problems are unchanged from those recognised at the turn of the century by German surgeons whose accounts have been summarised by Pontius, Clagett and McDonald (1959). Other American reports are from Haas (1914), Murphy (1916), Moschowitz (1918), Wilensky and Samuels (1926), Siler (1942) and Maier (1947). In 1935 Brock recorded an example of typhoid chondritis. It is significant that while the earlier papers are concerned with specific infections (tuberculosis, typhoid, actinomycosis), later ones refer increasingly to low-grade pyogenic infection.

The case histories of two patients are presented. They are in no way unique but they show the important features likely to be encountered in the condition today.

\section{CASE REPORTS}

Case 1-A twenty-year-old male Cypriot underwent operation for the correction of pulmonary stenosis with atrial septal defect. Operation was done under profound hypothermia (Drew

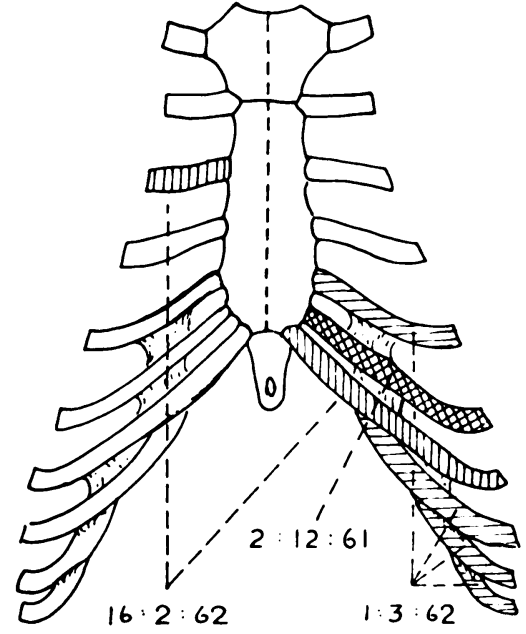

FIG. 1

Case 1-The excised costal cartilages are shaded. The dates on which the resections were performed are indicated. technique) in October 1961 by Mr Vernon Thompson. The exposure was through a median sternotomy. The operation and the period immediately after operation were uneventful, but on the seventeenth day after operation the patient complained of severe pain over the precordium and a small collection of pus discharged from the central part of the wound. The temperature had been slightly elevated for a few days (99-100 degrees Fahrenheit). Culture of the pus yielded a scanty growth of pseudomonas pyocyanea sensitive to a wide range of antibiotics.

Despite chemotherapy and blood transfusions for progressive anaemia his condition deteriorated; he kecame emotionally disturbed from toxaemia and excruciating pain, the latter necessitating frequent injections of pethidine. It was apparent that costal cartilages and also, perhaps, the sternum were involved in the infective process. Six weeks after the first operation part of the wound was reopened and free drainage established. The culture of debris evacuated from over the sternum yielded a profuse growth of pseudomonas pyocyanea with a much reduced range of sensitivity to antibiotics (streptomycin, polymyxin B, neomycin and colomycin). These drugs were given in varying combinations during the next three months. Meanwhile, at three further operations, infected costal cartilages were resected subchondrally from sternum to bony rib-this included the whole costal arch on the left side (Fig. 1). The sternum was 
irregular and thickened anteriorly but did not sequestrate. Within four weeks of the last operation in March 1962, the wounds were healed (Fig. 2).

Comment-It was desirable to avoid further operative stress for as long as possible after the cardiac operation-these resections through hyperaemic and oedematous tissues are accompanied by considerable haemorrhage.

By resecting the diseased cartilage in staged operations, instability of the chest wall was avoided. The regeneration of costal cartilage was well shown on radiographs: such cartilage seems to be more calcified than usual (Fig. 3). The sternum did not undergo necrosis despite the fact it had been split to expose the heart.

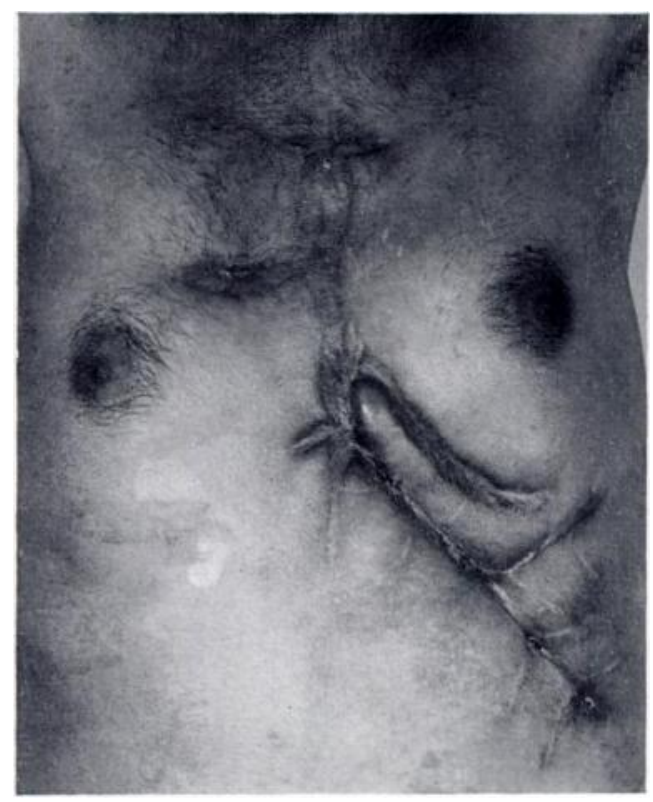

FIG. 2

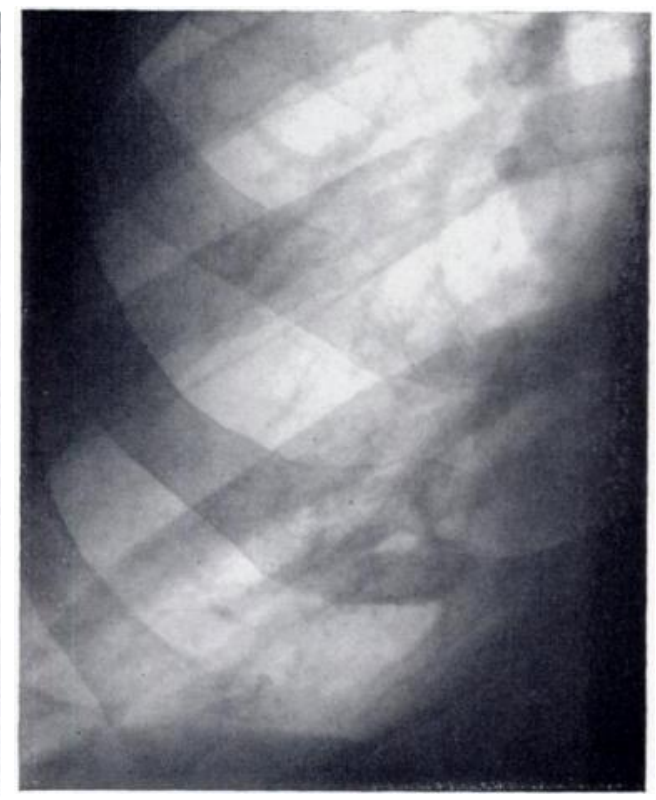

Fig. 3

Case 1. Figure 2-The appearance of the anterior chest wall when almost healed. Figure 3-Posteroanterior radiograph of chest showing regeneration of costal cartilages.

Case 2-In 1951 a fifty-eight-year-old man was admitted to the orthopaedic and accident department of The London Hospital. For the previous nine months he had been treated elsewhere for what had been diagnosed as septic arthritis of the right sternoclavicular joint. Initially he had been given penicillin, but because of persistent pain and tenderness, exploration and excision of the medial third of the clavicle had been done. A persistently discharging sinus had developed and when the patient was first seen at The London Hospital an abscess was developing over the lower end of the sternum.

The patient looked ill and had a temperature of 100 degrees Fahrenheit. There was a wet sinus four centimetres to the right of the suprasternal notch, and a swelling four centimetres in diameter, with multiple discharging ulcers, over the lower end of the sternum; both areas were tender, bluish and warm. The left shoulder was stiff and painful with a restricted range of movement. Radiographs of the chest, spine and left shoulder showed no foci of infection. A sinograph showed communication between the two areas extending throughout the whole length of the retrosternal space (Fig. 4). There were prolongations into the subcutaneous tissues overlying the sternum. Haemoglobin level was 9.6 grammes per 100 millilitres; the white cell count was 12,200 per cubic millimetre, with a normal differential count. Culture of the pus yielded a profuse growth of pseudomonas pyocyanea sensitive to streptomycin and chloramphenicol. 
96

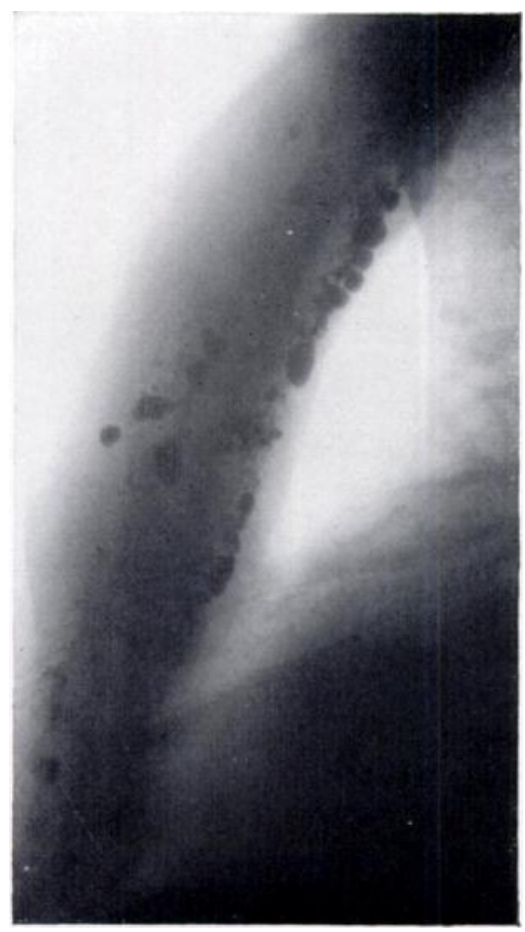

FIG. 4

Case 2-Sinograph showing retrosternal abscess with prolongations through the perforations in the sternum.

\section{R. A. ELSON}

The patient was thought to be suffering from a polyarthritis of rheumatoid type with an atypical distribution. It was thought that after operation the sternoclavicular region had become infected. Mr Vernon Thompson was asked to undertake the patient's further treatment.

The patient was given chloramphenicol and at operation in May 1951 the sternum was exposed through a vertical incision; its surface was soft, thickened, pitted, and there were sinuses perforating its whole thickness. Histological examination of fragments of this bone showed changes typical of chronic osteomyelitis. Free drainage was established and for a while the patient's condition improved. Soon, however, the pain and pyrexia increased again, and at a further operation three weeks later, a segment of sternum was removed. Some pockets of pus under tension, with fragments of necrotic cartilage, were evacuated.

Again the local and general condition improved and it was thought that the area might heal. The patient was sent for convalescence, but was readmitted urgently on account of renewed inflammation and profuse discharge of pus. A third operation was performed in October 1951, and a larger segment of sternum was removed, together with several fragmented and sequestrated costal cartilages (probably parts of the second to fourth on both sides). Culture at this time showed that the pseudomonas was sensitive only to streptomycin and only slightly sensitive to this drug. There was no improvement after operation and, at a further exploration, sinuses were traced to the lower end of the sternum on the right, and the whole costal arch was excised subperichondrally on this side. Three months later the left costal arch was found to be involved as well and was resected together with the whole lower part of the sternum. Finally, after another four months, a residual sinus at the upper end of the bone was explored and the first left costal cartilage removed (Fig. 5). The history since the onset of symptoms had now lasted twenty-three months. At one stage during this period temporary enlargement of the liver occurred and amyloid disease was feared; fortunately no other manifestation of this condition appeared.

After the last operation there was immediate improvement, although for nearly two more years there was one tiny sinus which discharged intermittently a little. The patient was seen at intervals of a year until he died suddenly from cardiac infarction in November 1960.

Comment-Probably the original condition was rheumatoid arthritis accompanied, as is usual, by a reduced resistance to bacterial infection. At the first operation, during exploration, the adjacent first costal cartilage was, perhaps, damaged. Had the true nature of the complication been recognised, the extensive spread of disease

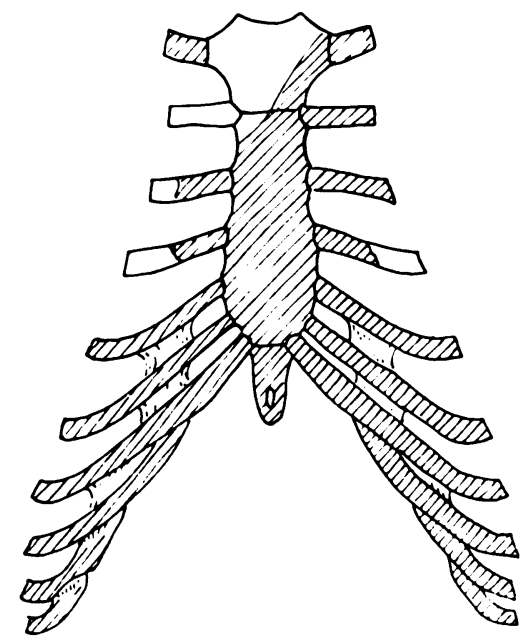

FIG. 5

Case 2-State in June 1952. The excised costal cartilages are shaded. 
might have been avoided by immediate excision of this one cartilage. The osteomyelitis of the sternum afforded a ready pathway for spread of infection to all the costal cartilages.

\section{DISCUSSION}

Incidence and etiology-Costal chondritis is uncommon; only two cases have been treated at The London Hospital during the last ten years compared with 663 of osteomyelitis affecting other bones. The largest published series, that from the Mayo Clinic (Pontius et al. 1959). comprised only seventeen cases.

Today the disease in its most troublesome form is a suppurative infection caused by organisms of a low-grade virulence. These succeed in establishing themselves only after cartilage necrosis has been produced by mechanical trauma, ischaemia, infection by more virulent organisms or poisoning by the toxins in exudates from neighbouring infective processes.

Injury to the costal cartilages may occur from penetrating wounds of the anterior chest wall or during operations in this vicinity. Bacteria reach the costal cartilages through such wounds, or by direct spread and the blood stream. The perichondrium becomes infected and this may cause local death of cartilage. If an abscess forms and drains to the exterior, a portal of entry for other organisms is established. The necrotic area of cartilage becomes colonised so that there is progressive sequestration of cartilage with continued infection.

Infection may occur from direct spread from lymph nodes of the internal mammary chain or from the lung, pleura, mediastinum or sternum. Sternal osteomyelitis is often associated with costal chondritis; but the bone does not so often undergo massive necrosis and sequestration.

Other infecting organisms-Before the introduction of specific anti-tuberculous drugs. tuberculosis accounted for most cases. The condition described graphically by Moschowitz (1918) resembles that in our cases. Secondary infection of cold abscesses was common.

Typhoid osteomyelitis was studied by Murphy (1916) who found that of 533 such infections seven involved the sternum and 135 the ribs; many of the latter affected the anterior end. Chondritis of the first costal cartilage was the presenting feature in a case of paratyphoid infection recorded by Brock (1935).

Other specific infections are blastomycosis (Maier 1947), actinomycosis (Maier 1947. Moschowitz 1918) and corynebacteria (Pontius et al. 1959).

At present the commonest infecting organism seems to be pseudomonas pyocyanea-this was so in the Mayo Clinic series and also in our two cases. Other pyogenic bacteria which may be primary infecting agents are staphylococcus aureus, pneumococcus, bacterium coli, haemolytic and non-haemolytic streptococci.

Pathological anatomy-The perichondrium is vascular and resists necrosis unless severely traumatised. New cartilage regenerates from it after subperichondral resection of necrotic cartilage. No regeneration occurs from the chondrocostal junction except in children where this is the growing end of the costal cartilage, nor from islands of cartilage left behind by incomplete resection (Haas 1914). Haas pointed out that under the microscope no clear demarcation between perichondrium and its underlying cartilage can be seen, although a plane of cleavage can be developed surgically. Because there are no blood vessels in cartilage the chondrocytes depend for their nutrition on diffusion of metabolites from this investing membrane. Toxins or ischaemia can easily undermine the viability of the chondrocyte.

The anatomy and relations of the costal cartilages are important for a clear understanding of the problems of treatment. The upper six costal cartilages are separate structures, but the seventh to the tenth constitute a branching system; it forms that part of the inferior costal margin which extends from the xiphoid process to as far laterally as the sharp upward sweep of the lower ribs. Infection of any one of these communicating cartilages can spread directly 
to involve the whole branch. Further, spread from one branch to that on the opposite side can occur by way of the xiphoid process, the lower half of which remains cartilaginous until old age (Siler 1942). Partial removal of the branch system must leave some areas of the remaining cartilages exposed and denuded of their nourishing perichondrium so that continuation of the necrosis is most likely.

Diagnosis and differential diagnosis-Inflammation of the chest wall after penetrating injury or surgical operation in the region of the costal cartilages should suggest the possibility of costal chondritis, especially if there is severe pain.

Closed infection in the absence of demonstrable suppuration may appear similar to Tietze's syndrome (chondropathia tuberosa), but bacterial infection is more likely to cause pyrexia and leucocytosis (Kayser 1956). Frank abscess formation must be differentiated from pus tracking round an intercostal space from the vertebrae, empyema necessitatis, or breast infections. Sinography with lipiodol may be of great value (Brown and Trenton 1952).

Complications-Infection often spreads to other costal cartilages, the sternum, sterno-clavicular or sterno-costal joints. Retrosternal abscess is dangerous; it is commoner after blood-borne infections of the sternum (Maier 1947). The pleural cavity or mediastinum can be involved. Amyloid disease from prolonged sepsis is mentioned in earlier reports.

Mortality - In cases where the sternum was involved, Siler (1942) recorded the heavy mortality of 27 to 50 per cent, showing how serious the disease could become without early adequate treatment. Anaemia, weakness from pain and toxaemia and amyloid disease may make operation hazardous.

Prophylaxis-All penetrating injuries in this area should be explored for damage to the costal cartilages. Any areas stripped of perichondrium should be covered by healthy muscle-the external intercostal muscle can be detached from one of its margins and reflected over such bared cartilage.

Care should be taken during operations in which the cartilages are prone to injury. In particular this applies to radical mastectomy, upper abdominal incisions (especially if draining infected material) and intrathoracic operations. Splitting the xiphoid process in a median upper abdominal incision does not appear to be dangerous in this respect (Siler 1942). Treatment-Before cartilage necrosis has occurred, adequate antibiotic therapy can cure this disease. If necrosis has occurred, the antibiotic usually controls the spread of cellulitis, but not the formation of a localised abscess or a smouldering chronic inflammatory mass: in these cases resection of the affected costal cartilage should be performed as soon as possible. This may be accomplished through a transverse incision over the cartilage concerned, the perichondrium is incised and stripped off with the rugine. It is easy to bite into the soft cartilage so that fragments of necrotic tissue are left behind to perpetuate the infection (perichondrium is more difficult to strip from cartilage than periosteum from bony rib). The excision must be total-that is, from sterno-costal joint medially to raw healthy bony rib laterally. If pus is present the wound should be drained: secondary infection is unlikely, if all dead tissue has been removed. The necrotic cartilage appears yellow, opaque and lustreless.

When the involved cartilage communicates directly with those forming the costal margin the whole branch system must be excised if cure is to be certain. However, if the necrosis is clearly localised or if the patient's general or cardio-respiratory condition preclude an extensive procedure, local resection may be attempted by the technique recommended by Maier (1947), in which the stumps of cartilage are trimmed back within their tubes of perichondrium. The latter are then oversewn so that the cartilage is completely covered. Care must be taken not to elevate the perichondrium from the cartilage stump during the removal with rongeurs of the last few millimetres. When an extensive resection is necessary, especially if the sternum is involved, the procedure may be performed in two or more stages so that instability is avoided. 
It is interesting to contrast this operation with that sometimes performed in osteomyelitis when local sequestrectomy results in healing; in costal chondritis, the whole cartilage is the "sequestrum" (Brown and Trenton 1952).

In specific infections resection is performed under an appropriate antibiotic cover. Adequate blood replacement should be ensured, since these procedures through oedematous hyperaemic tissue may prove very haemorrhagic. Care should be taken to avoid damage to other costal cartilages, the pleura, perichondrium and internal mammary vessels. The perichondrium should be traumatised as little as possible, so as to ensure cartilage regeneration and recovery of chest stability, especially when an extensive resection is necessary.

\section{SUMMARY}

1. Two cases of costal chondritis are presented. The special features and treatment of this condition are described and the literature is reviewed.

2. Early diagnosis and energetic treatment of costal chondritis obviates serious morbidity. 3. Treatment with antibiotics is of value in curing the condition before cartilage necrosis has occurred and in controlling the spread of infection to neighbouring tissues.

4. Excision of all necrotic cartilage is essential for cure, if cartilage necrosis has occurred.

5. Secondary infection with organisms of low virulence, notably pseudomonas pyocyanea, is nowadays the commonest cause of chronicity.

I am grateful to $\mathrm{Mr}$ Vernon C. Thompson for his permission to publish these cases.

\section{REFERENCES}

Brock, R. C. (1935): Post-Typhoid Chondritis with Abscess Formation. British Journal of Surgery, 23, 231. Brown, R. B., and Trenton, J. (1952): Chronic Abscesses and Sinuses of the Chest Wall. Annals of Surgery, 135, 44.

HaAs, S. L. (1914): Regeneration of Cartilage and Bone with a Special Study of These Processes as They Occur at the Chondrocostal Junction. Surgery, Gynecology and Obstetrics, 19, 604.

Kayser, H. L. (1956): Tietze's Syndrome-A Review of the Literature. American Journal of Medicine, $21,982$.

MaIER, H. C. (1947): Infections of the Costal Cartilages and Steriium. Surgery, Gynecology and Obstetrics, 84, 1038

Moschowitz, A. V. (1918): The Treatment of Diseases of the Costal Cartilages. Annals of Surgery, $68,168$.

Murphy, J. B. (1916): Bone and Joint Disease in Relation to Typhoid Fever. Surgery, Gynecology and Obstetrics, 23, 119.

Pontius, J. G., Clagett, O. T., and McDonald, J. R. (1959): Costal Chondritis and Perichondritis. Surgery, 45, 852.

Siler, V. E. (1942): Acute Costal Chondritis Associated with Sternal Osteomyelitis. Surgery, 12, 407.

Wilensky, A. O., and Samuels, S. S. (1926): Osteomyelitis of the Sternum. Annals of Surgery, 83, 206.

VOL. 47 B, NO. 1. FEBRUARY 1965 